\title{
Diversity Breeding Program on Common Bean (Phaseolus vulgaris L.) Targeting Rapid Cooking and Iron and Zinc Biofortification ${ }^{\dagger}$
}

\author{
Clare Mukankusi 1,** Wallace A. Cowling ${ }^{2}$, Kadambot H.M. Siddique ${ }^{2}$, Li Li $^{3}$, Brian Kinghorn ${ }^{4}$ \\ and Jean Claude Rubyogo ${ }^{1}$ \\ 1 Alliance of Bioversity International and International Center for Tropical Agriculture (CIAT)-Pan Africa \\ Bean Research Alliance (PABRA), P. O. Box 6247 Kampala, Uganda, P O. Box 823 - 00100, Nairobi, Kenya; \\ j.c.rubyogo@cgiar.org \\ 2 Institute of Agriculture, The University of Western Australia (UWA), M082, Perth WA 6001, Australia; \\ wallace.cowling@uwa.edu.au (W.A.C.); kadambot.siddique@uwa.edu.au (K.K.M.S.) \\ 3 Animal Genetics and Breeding Unit, University of New England, Armidale NSW 2351, Australia; \\ 1li4@une.edu.au \\ 4 School of Environmental and Rural Science, University of New England, Armidale NSW 2351, Australia, \\ Brian.Kinghorn@une.edu.au \\ * Correspondence: c.mukankusi@cgiar.org \\ + Presented at the third International Tropical Agriculture Conference (TROPAG 2019), Brisbane, Australia, \\ 11-13 November 2019.
}

Published: 8 April 2020

\begin{abstract}
Common bean (Phaseolus vulgaris L.) is a major component of agricultural systems and diets of the urban and rural populations of East and Central Africa, providing Fe and Zn essential to the health and well-being of African women and children, and protein essential for the entire household. However, bean consumption is limited by constraints such as long cooking time (CT). Cooking demands large amounts of water, fuel and time. It has negative effects on the environment, livelihoods, security and health. Genetic variability in cooking time is documented. Recent development of new breeding methods based on pedigree and genomic selection together with optimal contribution selection (OCS) offers an opportunity to accelerate breeding for rapid CT and higher Fe and Zn grain content. Genotypic and phenotypic data of an African diversity pool, representing key bean market classes, were used to generate genomic estimated breeding values (GEBVs) for grain yield, CT, Fe and Zn. GEBV's were weighted to maximise the desired outcome in an economic index. From 161 candidate bean genotypes with GEBVs, 67 were chosen for 80 matings within six major grain market classes. An additional 22 breeder nominated matings were included. The predicted outcomes in the first cycle showed a major improvement in population mean for index (+286.77 US\$/ha), 6.2\% increase in GY and 7.3\% reduction in CT, with an achieved increase in population co-ancestry of 0.0753 . A $30 \%$ reduction in the mean population CT and improved Fe $(15 \%)$ and $\mathrm{Zn}(10 \%)$, is expected after 5 cycles of annual recurrent selection.
\end{abstract}

Keywords: common bean; cooking time; iron; zinc;; genomic estimated breeding values; optimal contributions selection 